\title{
Neural Growth Into A Microchannel Network: Towards A Regenerative Neural Interface
}

\author{
Paul A. Wieringa, Remy W. F. Wiertz, Joost le Feber and Wim L. C. Rutten, Member, IEEE
}

\begin{abstract}
We propose and validated a design for a highly selective "endcap" regenerative neural interface towards a neuroprosthesis. In vitro studies using rat cortical neurons determine if a branching microchannel structure can counter fasciculated growth and cause neurites to separate from one another, allowing for greater selective contact. Initial studies find that narrower branching microchannels achieve improved neurite separation. Electrical stimulation of neurites within microchannels is possible, as is recording of neurite action potentials with the microchannels acting as electrical signal amplifiers.
\end{abstract}

Index Terms - Neural interface, tissue engineering, regenerative electrode, bidirectional communication interface.

\section{INTRODUCTION}

$\mathrm{T}$ here has been much progress in neuroprosthetic limb development, most notably brain-machine interfaces and the development of myoelectrically controlled prosthetic arms[1]-[3]. However, both neural interfacing approaches have limited control over neuroprosthesis and also provide a small degree or no neural feedback to the user[2]; this is cited as a requisite toward improved acceptance and usability of prosthetic limbs in the daily lives of patients[4]. The work presented here is an initial investigation into a peripheral nervous system (PNS) interfacing approach proposed to address issues of alternate interfacing strategies while providing improved control and feedback for a neuroprosthetic device.

Afferent and efferent PNS neurons provide sensory feedback and motor control, respectively, to and from the central nervous system. Both neuron types are bundled into fascicles, which then bundle together to form the nerves found within the body[5]. Many PNS interfaces insert electrodes into nerves, but the spatial distribution of the bundled neurons limit the ability of an interface to reliably establish selective electrical contact (for recording and/or stimulation) with individual neurons. This compromises the control and feedback available for neuroprostheses $[6,7]$.

An alternative relies on the regenerative properties of PNS neurons. If a nerve bundle is severed, neurites regrow from the proximal nerve stump in an attempt to find appropriate innervation targets[4]. Sieve electrodes take advantage of this mechanism, with a disc-like structure placed in a nerve

Manuscript received January 12th, 2009. All authors are with the Biomedical Technology Institute at the University of Twente, 7522 NB Enschede, NL (P. A. Wieringa, corresponding author: +31 (0)53 489 2760; fax: +31 (0)53 489 2287; e-mail: P.A.Wieringa@ewi.utwente.nl). transection between the proximal and distal ends of the stump. Neurites grow into the distal stump via micron-sized thruholes in the disc; electrical contact is established by a circumferential electrode around perimeter of the thru-hole. Successful neural ingrowth only occurs for hole diameters of approximately $50 \mu \mathrm{m}$, relatively large compared to axon diameters of 0.5 to $4 \mu \mathrm{m}[6]$. This still leads to many neurons being associated with one thru-hole, which is further complicated by neurites' use of preexisting axons as scaffolds to guide growth in a bundled, or fasciculated, manner. Although an improvement, this approach still does not achieve the ideal of 1-to-1 electrode-to-neuron contact.

Proposed here is a three dimensional endcap that is placed on the end of the proximal nerve stump at the site of amputation. In lieu of thru-holes are a series of microchannels into which neurites will grow. Microchannel size is kept initially large to encourage neural ingrowth, but these microchannels undergo repeated bifurcations as they proceed further into the endcap. The bifurcating microchannels are proposed to promote separation of the ingrowing nerve bundle, with enough bifurcations to eventually have individual neurons in individual microchannels. Neurite growth can then be guided toward individual electrode sites, creating high selectivity for effective neural recording and stimulation.

This in vitro study attempts to verify the concept of using bifurcating microchannels to promote a separating pattern of neural ingrowth, with the examination of different microchannel sizes toward an optimal design. Also examined is the ability to electrically record and stimulate neurites in microchannels.

\section{Methodology}

\section{A. Culturing Device}

To investigate neurite growth in bifurcating microchannels, a PDMS (Sylgard) microfluidic device was micromolded on a silicon wafer to create a cast consisting of two large open chambers, defined by SU8 and post-cast cutting, and of microchannels connecting the two chambers, defined across the 'Bridge'(see Fig. 1) by reactive ion etching (RIE) the silicon wafer[8]. The resulting PDMS cast was reversibly sealed, microchannels facing down, onto a clean, dry glass substrate that was previously coated for 2 hours with a 50 $\mathrm{ug} / \mathrm{ml}$ polyethyleneimine (PEI) solution (Sigma-Aldrich) to promote neuronal cell adhesion to the substrate[9]. This creates the culturing device pictured in Figure 1 with an open 'Origin' chamber and 'Target' chamber connected via 
enclosed bifurcating microchannel networks, with all sections of the device having a culturing surface suitable for optical observation.

The microchannels had a rectangular cross section with a single 'Primary' channel bifurcating into two 'Secondary'

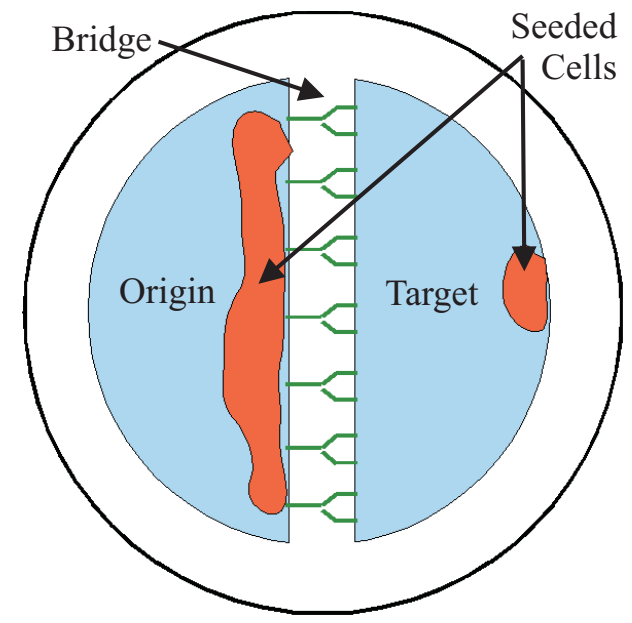

Fig. 1. The culturing device with the Origin chamber seeded with cells and the Target chamber with 'Target' cells. The device has a $22 \mathrm{~mm}$ outer diameter, a $16 \mathrm{~mm}$ inner diameter and a Bridge width/channel length of 1 $\mathrm{mm}$. PDMS cast height is $4 \mathrm{~mm}$, resulting in $400 \mathrm{uL}$ per chamber for culture medium..

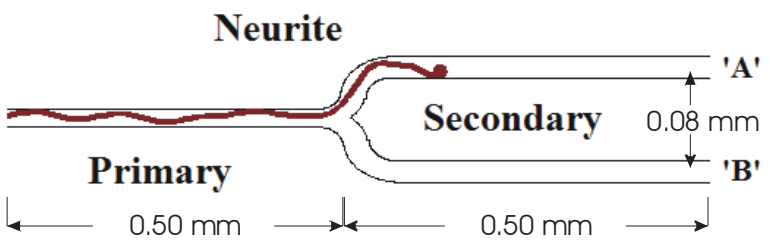

(a)

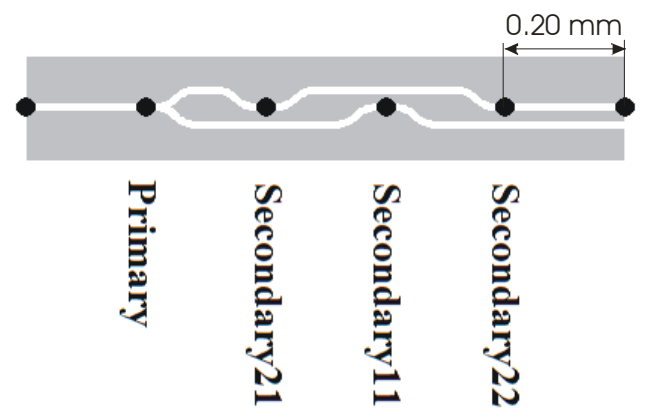

(b)

Fig. 2. a) Bifurcating microchannel design with a pioneering neurite growing down the primary channel and selecting a secondary channel. Channels widths of 10 and $20 \mu \mathrm{m}$; b) A modified microchannel for MEA layout, shown with and electrode labels and electrode positions shown $200 \mu \mathrm{m}$ apart to cover the channel length of $1 \mathrm{~mm}$. Channel widths of 2.5, 5, 10 and 20 $\mu \mathrm{m}$.

channels (Figure 2a). Channel height was $5 \mu \mathrm{m}$ and widths of $10 \mu \mathrm{m}$ and $20 \mu \mathrm{m}$ were optically examined. Electrical examination took place by substituting the glass substrate with "200/30-i.R.-Ti-w/o" multi-electrode array (MEA) from MultiChannel Systems (MCS) GmbH, Germany. The MEAs consist of planar glass substrate incorporating 60 electrodes, $30 \mu \mathrm{m}$ in diameter and $200 \mu \mathrm{m}$ apart in an $8 \mathrm{x} 8$ grid, with leads insulated by an optically clear silicon dioxide layer. The microchannels were redesigned to accommodate the MEA electrodes and the PDMS cast was carefully aligned on top of the electrode array with one electrode interfacing with one microchannel segment(Figure 2b), making possible electrical measurement and stimulation of neurites within the microchannels as well as interfacing with cells in the two chambers. Electrodes within the microchannels were partially obscured by the PDMS cast, leaving an active electrode area with the width of the microchannel and approximately $30 \mu \mathrm{m}$ in length. For this study, microchannel widths of 2.5, 5, 10 and $20 \mu \mathrm{m}$ were evaluated.

\section{B. Cell Preparation}

The dissociated cortical neurons used were taken from 1-3 day old postnatal Wistar rats. Cortical cell cultures ensure low Glial cell content, allowing for improved observation of neuronal growth. Briefly, rats were decapitated and cortices were dissociated from surrounding tissue. The cortical tissue pieces were placed in a tube of $3 \mathrm{ml}$ of $\mathrm{R} 12$ culture medium[10]. $1 \mathrm{ml}$ of trypsin was added, incubated for 45 minutes at $37^{\circ} \mathrm{C}$, and the supernatant was removed and replaced by $0.5 \mathrm{ml}$ of soybean trypsin inhibitor, $100 \mu \mathrm{l}$ of DNAse, and enough R12 to obtain total volume of $1 \mathrm{ml}$. This was mechanically dissociated and the supernatant, containing the successfully dissociated cells, was removed and centrifuged at $1200 \mathrm{rpm}$ for 5 minutes. The resulting pellet of dissociated cells was resuspended to a achieve a relatively high cell concentration (approx. $2 \times 10^{6}$ cells $/ \mathrm{ml}$ ). This cell suspension was seeded along the dividing bridge of the Origin chamber resulting in a mono layer of $\sim 4000$ cells $/ \mathrm{mm}^{2}$, with a small drop placed in the Target chamber to act as a distant target tissue (Figure 1). Devices were placed in an incubation chamber at $37^{\circ} \mathrm{C}$ at $5 \% \mathrm{CO}_{2}$, with $\mathrm{R} 12$ medium refreshed every 3 days.

\section{Visual Examination}

Observation of neural growth was performed by labeling
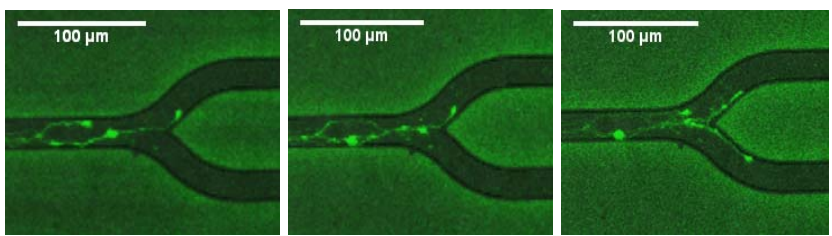

Fig. 3. An example sequence of recorded images: neurites at the decision branch point of a $20 \mu \mathrm{m}$ wide microchannel. Images taken $1 \mathrm{hr}$ apart.

neurons with CellTracker green $(12.5 \mu \mathrm{M})$ from Invitrogen. A Carl Zeiss LSM 510 was set for FITC labeling and was fitted with an environmental chamber and an automated stage for time series image recording of neuronal growth at multiple locations within the culturing device (example images, Figure 3 ). A single culture device was evaluated 2 days after initial cell seeding for three days at $10 \mathrm{x}$ magnification and 15 
minutes between images, recording growth in $10 \mu \mathrm{m}$ wide and $20 \mu \mathrm{m}$ wide microchannels (20 of each size). The resulting videos were manually evaluated for: a) number of ingrowing neurites; b) number of channels experiencing separation.

\section{Electrical Examination}

Electrical recording and stimulation used the MCS 1060BC preamplifier and an NI6071E acquisition card (National Instruments), sampling at $16 \mathrm{kHz}$, in conjunction with associated MCS software and a customized Labview application. A 2 week-old culture was examined with 2 microchannels of each width: $2.5,5,10$ and $20 \mu \mathrm{m}$. Action potential (AP) recording used a detection threshold previously established to eliminate false positive detection in an open culture (no PDMS microchannels). This threshold is calculated as 5.5 times the estimated RMS noise value. Noise estimation was taken as the weighted average of the previous noise estimation and the RMS calculated over the current 10 ms window. Since the experimental setup reduced electrode area by PDMS placement, thus theoretically translating to increased thermal noise, the measurement setup was first validated by examining the signal-to-noise ratio (SNR) to ensure neurite AP recording was still possible[11].

Point process analysis was employed to track individual AP propagation by virtue of the multiple electrode sites within the bifuricating microchannel. To isolate individual AP events, additional detection criteria eliminated compound APs resulting from the multiple neurites growing within a microchannel. After the Primary electrode (Figure 2b) detected an AP, a trailing $20 \mathrm{~ms}$ window was employed to find APs occurring at all electrode sites within the microchannel. The occurrence of these APs was accumulated into a latency histogram with a bin size of $0.0625 \mathrm{~ms}$. For every AP detected at the Primary electrode, subsequent AP latency times were accumulated into a final histogram. Stimulation studies applied a 20 uA biphasic current pulse, 200 us per phase.

\section{RESULTS \& DISCUSSION}

\section{A. Neurite Growth}

Table 1 shows the average number of neurites is larger in wider channels than in narrow channels. However, the current experimental approach makes it impossible to adjust these values for inconsistencies in cell seeding density. Although indicative of the degree of successful neurite ingrowth, nothing conclusive can be said about the influence of channel size in this respect.

Growth was typically observed to begin with one or two 'pioneering' neurites, followed by later arriving neurites. At the bifurcation, the pioneering neurites chose one secondary path (designated 'A', see Figure 2a). The later neurites often followed, though some later neurites would select secondary path 'B'. At this point, a microchannel is said to have experienced separation. Observations so far find no pattern between 'separation' growth behaviour and the amount of ingrowth present within the channel, implying such growth behaviour is independent of cell seeding density(data not shown). Instead, the separation behaviour appears to be dependent on microchannel size (Table 1).

The narrow channels show increased 'separation'. This is attributed to the random exploratory growth of a neurite

TABLE I

STATISTICAL INFORMATION ON NEURITE GROWTH

\begin{tabular}{|c|c|c|}
\hline \multirow{2}{*}{ Property ${ }^{\mathrm{a}}$} & \multicolumn{2}{|c|}{ Per Channel Width } \\
\hline & $10 \mu \mathrm{m}$ & $20 \mu \mathrm{m}$ \\
\hline $\begin{array}{c}\text { Average \# of Neurites } \\
\text { (Std. Dev.) }\end{array}$ & $\begin{array}{c}5.25 \\
(3.22)\end{array}$ & $\begin{array}{c}7.95 \\
(5.61)\end{array}$ \\
\hline \multicolumn{3}{|l|}{ Growth Behaviour: } \\
\hline$\%$ Not Separated & 20 & 25 \\
\hline$\%$ Separated & 75 & 60 \\
\hline$\%$ No Growth & 5 & 15 \\
\hline \multicolumn{3}{|l|}{ Neurite Turning: } \\
\hline Absolute \# of Turns & 13 & 48 \\
\hline Adjusted \# of Turns ${ }^{b}$ & 0.167 & 0.339 \\
\hline
\end{tabular}

growth cone, which would deviate from the preceding neurite bundle but would generally return. If the random exploration occurred near the bifurcation, this neurite would become segregated in the alternate secondary channel and separated growth would proceed. It is suggested this is enhanced for smaller microchannels, as identical random deviations in a smaller space have a greater chance of resulting in segregation.

An additional observation in Table 1 includes neurites performing a $180^{\circ}$ turn while growing, attempting to exit via the channel entrance. This occurred much less for narrower channels, even when the number of turns per channel was

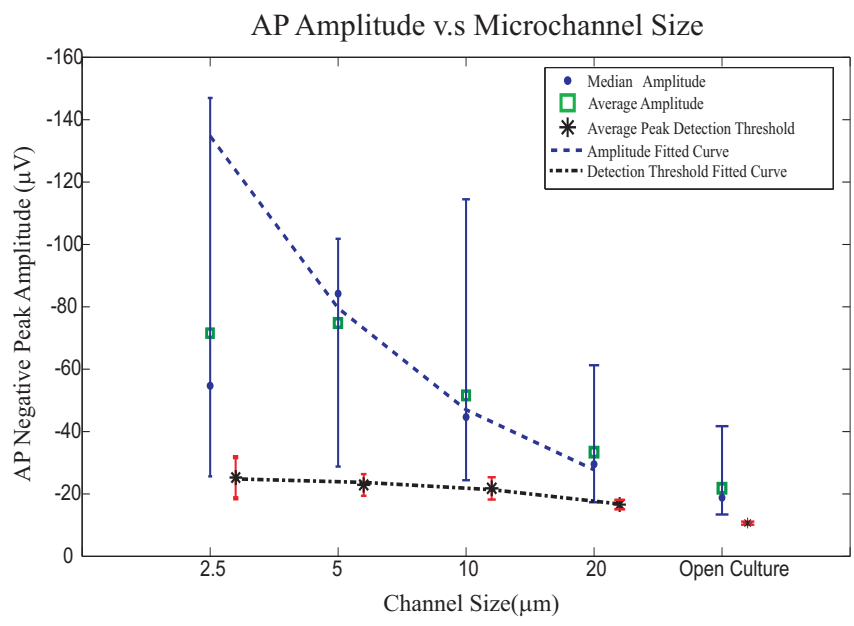

Fig. 4. A comparison of AP amplitudes and AP detection levels for different microchannel sizes. Amplitude data includes the mean (O), median $\left({ }^{\bullet}\right)$ and errorbars showing the $5^{\text {th }}$ to $95^{\text {th }}$ percentile amplitude range. The detection level is represented by the mean value $(*)$ and the standard deviation. The median amplitude log-log curve fitting has an $\mathrm{R}^{2}=0.985$. The mean detection threshold fitted curve has an $\mathrm{R}^{2}=0.9761$. Microchannel widths examined are $2.5,5,10$, and $20 \mu \mathrm{m}$ as well as electrodes located in 'Open Culture'.

divided by the number of neurites per channel to compensate 
for ingrowth dependencies (see Table 1:'Adjusted \# of Turns'). Based on visual observations, this difference is attributed to the reduced available space for neurites to complete a turn. Neurites would often appear to make a turning attempt, only to be corralled down the length of the microchannel when the apparent turning radius exceeded the microchannel width.

\section{B. Electrical Recording}

The electrical measurements performed recorded the activity of network bursts, seemingly random electrical

AP Propagation Latency Histogram
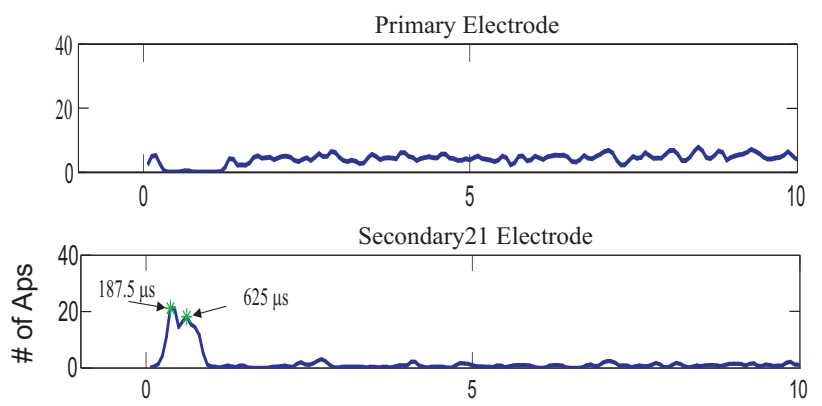

Secondary 22 Electrode

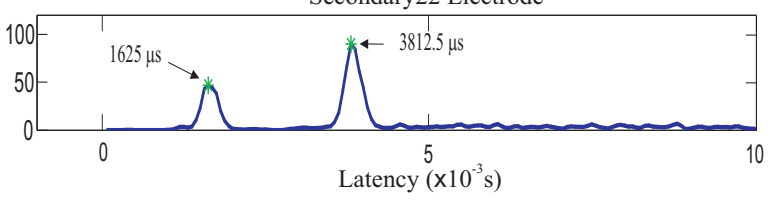

Fig. 5. Point process analysis of AP propagation through the microchannel, represented as a histogram of \# of Recorded APs with respect to latency time of AP occurence. Refer to Figure $2 b$ for designated electrode placement within the microchannel. Note the peaks of recurring AP events(arrows).

activity which initiates in a particular region of the neuronal culture and subsequently spreads to the rest of the culture via the synaptic connects that have been formed. This can be spontaneous or the result of an AP triggered by an electrode, which then propagates throughout the culture. The electrical measurements reveal that the setup is capable of effectively recording AP events, as seen in Figure 6a.

The amplitude distribution of recorded APs per channel size, shown in Figure 4 represented as the $5^{\text {th }}$ to $95^{\text {th }}$ percentile, is sufficiently above the mean detection threshold (*). These results also support the theory of signal amplification of APs recorded within a microchannel with sufficiently reduced extracellular volume relative to the neurite diameter[12].

Curve fitting of the detection threshold with respect to channel size (thus reduced electrode area) shows an inversely linear relationship. This implies that electrode noise holds the same relationship with channel size. Comparatively, curve fitting of the amplitude median values fits well with a log-log model with a slope (-0.756) similar to that derived from [12] (0.783 ). However, the $2.5 \mu \mathrm{m}$ wide channels show signal amplification lower than expected. This indicates a potential optimization around $5 \mu \mathrm{m}$ that strikes a balance of signal/noise amplification as well as unknown factors, such as sufficient channel size to permit neural ingrowth/maturation.
Successful tracking of action potentials within the microchannel network was performed, as shown in Figure 5.
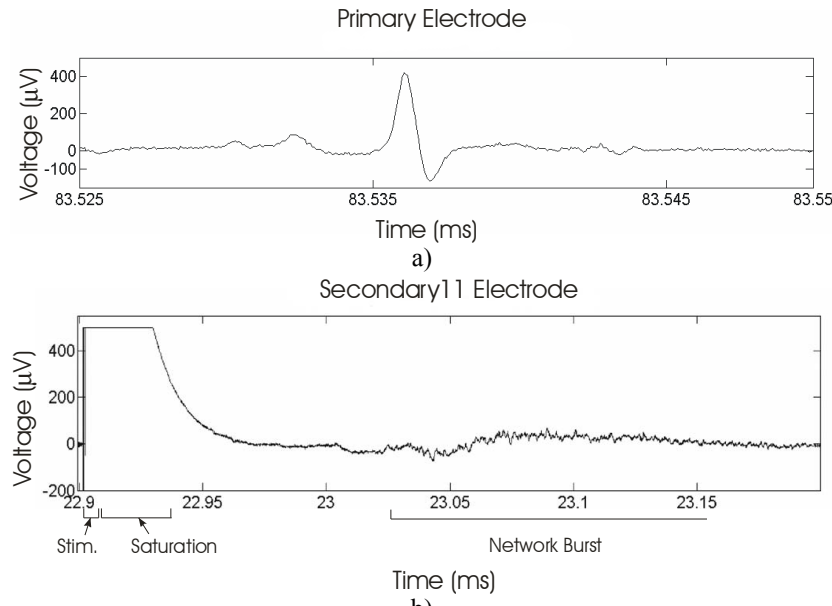

b)

Fig. 6. a) An example of an action potential recorded at the Primary Electrode of a $5 \mu \mathrm{m}$ wide microchannel; b) An example of a network burst resulting from stimulation at the Secondary11 Electrode within a $10 \mu \mathrm{m}$ wide microchannel. The recording was taken from the same Secondary11 Electrode site. Note the period of stimulation $(400 \mathrm{~s} \mu \mathrm{s})$ followed by a period of amplifier saturation and then the resulting network burst as it propagates back through the microchannel along ingrowing neurites. This data is collected directly after stimulation without relying on detection thresholds or other detection criteria.

The distinct histogram peaks of the point process analysis indicate that these AP events are highly repeatable. This implies the same AP is being recorded later in time as it propagates down the microchannel. Note also that following the initial AP detection at the Primary electrode (Time $0 \mathrm{~ms}$ of the $20 \mathrm{~ms}$ window of examination) there is a period of AP detection for approximately $1 \mathrm{~ms}$, corresponding well to the known refractory period of axons after experiencing an AP event.

In addition, since axons of different diameters have different diameters and, thus, different AP propagation velocities, differentiation between different-sized neurites based on their respective AP velocities should be possible[13]. Figure 5 shows two distinguishable peaks measured in the same channel and these peaks become further separated at an electrode further down the channel, indicating further velocity differentiation. This has direct implications for neural interfacing applications; by employing two or more electrodes within a microchannel one can differentiate signals even in the presence of fasciculated growth.

\section{Electrical Stimulation}

The application of a stimulus pulse to initiate an AP also proved successful, although immediate recording of any directly resulting APs was prevented by a stimulus artifact experienced simultaneously at all electrode sites. Successful stimulation was confirmed by a consistent occurrence of network bursts in the Origin chamber, resulting from triggered APs propagating out from the site of stimulation within the microchannel and into the neuronal culture (Figure 6b). This 
final result completes the proof-of-concept for bidirectional communication between electrodes and neurites within microchannels.

\section{CONCLUSION}

In vitro, neurons will grow into a network of microchannels and branching microchannels appear to promote neurite separation, with smaller channels increasing the probability of separation and reducing the probability of neurite turning. Electrical recording and stimulation are possible within microchannels, with electrical signal amplification occurring for smaller channels.

\section{REFERENCES}

[1] M. A. Lebedev, J. M. Carmena, J. E. O'Doherty, M. Zacksenhouse, C. S. Henriquez, J. C. Principe, and M. A. L. Nicolelis, "Cortical ensemble adaptation to represent velocity of an artificial actuator controlled by a brainmachine interface," J Neurosci, vol. 25, pp. 4681-4693, May 2005.

[2] T. A. Kuiken, P. D. Marasco, B. A. Lock, R. N. Harden, and J. P. A. Dewald, "Redirection of cutaneous sensation from the hand to the chest skin of human amputees with targeted reinnervation," Proc Natl Acad Sci U S A, vol. 104, pp. 20061-20066, Dec 2007.

[3] T. A. Kuiken, L. A. Miller, R. D. Lipschutz, B. A. Lock, K. Stubble_eld, P. D. Marasco, P. Zhou, and G. A. Dumanian, "Targeted reinnervation for enhanced prosthetic arm function in a woman with a proximal amputation: a case study," Lancet, vol. 369, pp. 371-380, Feb 2007.

[4] E. A. Biddiss and T. T. Chau, "Upper limb prosthesis use and abandonment: a survey of the last 25 years," Prosthet Orthot Int, vol. 31, pp. 236-257, Sep 2007.

[5] C. Ide, "Peripheral nerve regeneration," Neurosci Res, vol. 25, pp. 101-121, Jun 1996.

[6] X. Navarro, T. B. Krueger, N. Lago, S. Micera, T. Stieglitz, and P. Dario, "A critical review of interfaces with the peripheral nervous system for the control of neuroprostheses and hybrid bionic systems," J Peripher Nerv Syst, vol. 10, pp. 229-258, Sep 2005.

[7] W. L. C. Rutten, "Selective electrical interfaces with the nervous system," Annu Rev Biomed Eng, vol. 4, pp. 407-452, 2002.

[8] J. C. McDonald, D. C. Duffy, J. R. Anderson, D. T. Chiu, H. Wu, O. J. Schueller, and G. M. Whitesides, "Fabrication of microfluidic systems in poly(dimethylsiloxane)," Electrophoresis, vol. 21, pp. 27-40, Jan 2000.

[9] A. R. Vancha, S. Govindaraju, K. V. L. Parsa, M. Jasti, M. González-García, and R. P. Ballestero, "Use of polyethyleneimine polymer in cell culture as attachment factor and lipofection enhancer," BMC Biotechnol, vol. 4, p. 23, Oct 2004

[10] H. J. Romijn, F. van Huizen, and P. S. Wolters, "Towards an improved serum-free, chemically defined medium for long-term culturing of cerebral cortex tissue," Neurosci Biobehav Rev, vol. 8, no. 3, pp. 301-334, 1984.

[11] M. O. Heuschkel, M. Fejtl, M. Raggenbass, D. Bertrand, and P. Renaud, "A three dimensional multi-electrode array for multi-site stimulation and recording in acute brain slices," J Neurosci Methods, vol. 114, pp. 135-148, Mar 2002

[12] J. J. Fitzgerald, S. P. Lacour, S. B. McMahon, and J. W. Fawcett, "Microchannels as axonal ampliflers," IEEE Trans Biomed Eng, vol. 55, pp. 1136-1146, Mar 2008.

[13] D. K. Hartline and D. R. Colman, "Rapid conduction and the evolution of giant axons and myelinated fibers," Curr Biol, vol. 17, pp. R29-R35, Jan 2007. 